\title{
STAT6 attenuated murine acute lung injury through NLRP3/p38 MAPK/NF-kappaB signaling in macrophages
}

Lu Hu

Zhongshan Hospital Fudan University

Changzhou Shao

Zhongshan Hospital Fudan University

Linyue Pan

Zhongshan Hospital Fudan University

Zhilong Jiang ( $\square$ jiang.zhilong@zs-hospital.sh.cn )

Zhongshan Hospital Fudan University https://orcid.org/0000-0001-6217-5082

\section{Research Article}

Keywords: STAT6, acute lung injury (ALI), macrophage subtypes, NLRP3/p38 MAPK/NF-kappaB signaling

Posted Date: May 26th, 2021

DOI: https://doi.org/10.21203/rs.3.rs-438142/v1

License: @ (i) This work is licensed under a Creative Commons Attribution 4.0 International License.

Read Full License 


\section{Abstract}

Signal transducer and activator of transcription 6 (STAT6) is an intracellular transcription factor, it remained unclear whether STAT6 affects murine acute lung injury (ALI) through modulation of macrophage subtypes and NLRP3/p38 MAPK/NF-kappaB signaling. We in this study, intratracheal treated wild-type (WT) and STAT6-/- mice with $5 \mathrm{mg} / \mathrm{kg}$ LPS. Lung tissues and bronchoalveolar lavage (BAL) were collected 2 days after the treatment. The results showed that lack of STAT6 in STAT6-/- mice caused more severe lung inflammation, neutrophil influx, and the expression of TNF- $a$, IL- 6 and IL-1 $\beta$ in the inflamed lung tissues. Flow cytometry analysis showed Siglec F-CD206- biased polarization of M1 subtype macrophages in the LPS-treated STAT6-/- mice. In addition, lack of STAT6 increased the expression of NLRP3, p-p38 M APK, TNF- $a$, IL-1 $\beta$ and Calreticulin in the lung tissues of LPS-treated mice and STAT6-/- bone marrow-derived macrophages (BMDMs). However, Glibenclamide, PDTC and SB203580 effectively reversed the up-regulated pro-inflammatory cytokines in STAT6-/- BMDMs. Thereby, STAT6 defciency increased ALI severity, possibly through increasing polarization of M1 subtype macrophages and NLRP3/p38 MAPK/NF-kappaB signaling. NLRP3/p38 MAPK/NF-kappaB signaling may particiupate in the polarizatin of M1 subtype macrophages. Modulation of macrophages subtypes by molecular intervention of STAT6 signaling would be a promising therapeutic approach in the treatment of ALI.

\section{Inrtoduction}

STAT6 is an intracellular transcription factor and plays an important role in cell proliferation and differentiation [1, 2]. Recent studies showed that STAT6 is critically involved in asthma and lung fibrosis. Inhibition of STAT6 activity by inhibitory peptide markedely reduced the development of airway hyperresponsiveness and lung eosinophilia in mice following respiratory syncytial virus reinfectionin, associated with low production of IL-4 and polarization of alternatively activated macrophages (M2 cells) in the lung tissues [3]. In asthmatic mouse model, the expression of IL-4 and IL-13 is significantly increased, that activates downstream STAT6 signaling in eosinophils and promotes Th2 cytokine responses [4,5]. In addition, STAT6 is also involved in the suppression of lung fibrosis, because inhibition of STAT6 activity by STAT6 inhibitor AS1517499 significantly enhanced the development of lung fibrosis, the debilitating effects are associated with then reduced macrophage efferocytosis and increased proinflammatory cytokine expression, neutrophil recruitment into the lung tissues, but activation of STAT6 increased macrophage efferocytosis, improved lung inflammation resolution and attenuated lung fibrosis [6]. Because macrophage phagocytosis plays pivotal role in clearance of apoptotic neutrophils and debris in tissues. Efficent clear-up of the apoptotic neutrophils and debris facilitates the inflammation resolution and tissue repair after inflammation-induced tissues damage $[7,8]$. Therefore, STAT6 is an important intracellular trascrition factor in cells and critically involved in asthma and lung fibrosis.

Recent studies also showed the critical involvement of STAT6 in the development of acute lung injury (ALI) in mice and acute respiratory distress syndrome (ARDS) in patents $[9,10]$. For example, lack of STAT6 increases pancreatitis-associated lung injury and the expression of myeloperoxidase activity [11]. 
Additional study also showed that STAT6 participated in IL-4 biological function in murine ALI, because lack of STAT6 in macrophages abrogated the therapeutic effects of IL-4 [9]. In addition, STAT6 is also responsible for the therapeutic effects of MCTR1, an endogenously pro-resolution lipid mediator on ALI, because MCTR1 treatment up-regulated STAT6. However, STAT6 inhibitor AS1517499 abolished the therapeutic effects of MCTR1, indicating the beneficial role of STAT6 signaling in ALI [12]. Our previous study also showed that Calreticulin neutralizing antibody can suppress LPS-induced macrophage activation, but lack of STAT6 in macrophages induced resistance of macrophages in response to Calreticulin neutralizing antibody, further supporting the immune regulatory role of STAT6 in modulation of macrophage phenotypes and function [10].

A body of evidence indicated that STAT6 effectively drives the polarization of anti-inflammatory M2 cells. The biological function is closely related to the pro-allergic effects of STAT6 in asthma. In mice with ALI, the expression of TNF-a, IL-1 $\beta$ and IL- 6 were increased. Pyroptotic macrophages is thought a major source of IL-1 $\beta$. Under oxidative stress, NLRP3 (Nod-like receptor containing a pyrin domain 3 ) inflammasome in macrophages is activated to exclusively release a large amount of IL-1 $\beta$ and IL-18 from the pyroptotic macrophages $[13,14]$. NLRP3 inflammasome is composed of NLRP3, ASC (Apoptosisassociated speck-like protein containing a CARD) and pro-Caspase-1. The activated NLRP3 inflammasome can release active IL-1 $\beta$. LPS and other insultants are inducers of NLRP3 inflammasome, but that can be suppressed by IL-4 [15]. Though the expression of STAT6 and NLRP3 is affected in mouse model with ALI, it remains unclear whether LPS drive activation of NLRP3 inflammasome and macrophage pyroptosis through modulation of STAT6 signaling. To address this issue, we in this study, treated wild-type (WT) and STAT6-/- mouse model with LPS. The results showed that lack of STAT6 in STAT6-/- mice induced more severe ALI, associated with higher expression of NLRP3, p38 MAPK and proinflammatory cytokines than wild type mice with ALI. Because ATP-sensitive $\mathrm{K}(+)$ channel inhibitor Glibenclamide [16], PDTC and SB203580 are inhibitors of NLRP3, NF-kappaB and p-p38 MPK, we blocked the activities of NLRP3, NF-kappaB and p38 MAPK by these inhitors and the results showed that Glibenclamide, PDTC and SB203580 effectively attenuates LPS-induced macrophage pyroptosis and proinflammatory cytokine expression in vitro. Therefore, STAT6 signaling is protective in ALI. The beneficial effects were mediated through suppressing activation of macrophage pyroptosis via NLRP3/p38 MAPK/NF-kappaB signaling pathway.

\section{Methods}

\section{Mice and Treatment}

8-10 weeks old WT and STAT6-/- C57BL/ 6 male mice were intratracheal (i.t.) injected with $5 \mathrm{mg} / \mathrm{kg}$ lipopolysaccharides (LPS) from Escherichia coli 055:B5 (Sigma-Aldrich, St Louis, MO) for 2 days. The mice treated with PBS were used as controls. Bronchoalveolar lavage (BAL) and lung tissues were collected for analysis. All animals were housed and treated under the guidelines of the Institutional Animal Care and Use Committee of the Fudan University, Zhongshan Hospital in China. All experiments were approved by the committee and performed in the Zhongshan Hospital, Fudan University. 


\section{Culture and treatment of BMDMs}

Bone marrow cells were flushed from the femurs and tibiae of mice and cultured in RPMI1640 culture medium supplied with $10 \%$ fetal bovine serum (FBS) and $20 \%$ conditional media of NIH3T3 cells for 6 days to obtain bone marrow-derived macrophages (BMDMs). BMDMs from WT and STAT6-/- mice were pre-treated with $20 \mu \mathrm{M}$ NLRP3 inhibitor Glibenclamide, $20 \mu \mathrm{M}$ NF-kappaB inhibitor PDTC (Sigma, Saint Louis, Missouri) and 20 MM p38 MAPK inhibitor SB203580 (Cell Signaling Technology, Danvers, MA) for 1 $\mathrm{hr}$, and followed by stimulation with $500 \mathrm{ng} / \mathrm{ml}$ LPS for $24 \mathrm{hrs}$. The untreated or LPS alone treated cells were used as controls. Flow cytometry and immunostaining were used for analysis of cell activation and pyroptosis. The supernatants were collected for cytokine expression by ELISA assay.

\section{Western blot analysis}

The protein expression and activation of STAT6, p-p38 MAPK, ASC and NLRP3 in the lung tissues and cells were analysed by Western blot analysis. Primary antibodies included rabbit anti-total STAT6 (Bioss antibodies, Boston, MA), rabbit anti-ASC and rabbit anti-NLRP3 (Abcam, Cambridge, MA) and rabbit antip-p38 MAPK (Cell signaling technology, Danvers, MA). The anti-mouse glyceraldehyde-3-phosphate dehydrogenase (GAPDH) antibody was used as a loading control. Protein expression was quantitatively analysed on ImageJ software and data was presented as ratio of densitometric intensity of target protein to internal control GAPDH.

\section{Immunostaining assay}

The expression of p-STAT6 and NLRP3 in the lung tissues of mice were analysed by immunostaining assay. Briefly, the lung tissue sections were fixed with $4 \%$ paraformaldehyde. After incubation with $0.05 \%$ Triton X-100 and 10\% goat serum, the sections were incubated with primary antibody (dilution 1:200) for 3 hrs and followed by incubation with Cy3-conjugated anti-rabbit IgG for 1 hour. After washing with PBS for 3 times, the stained cells were visualized under fluorescence microscope.

\section{ELISA assay}

The concentration of TNF-a, IL-6, IL-1 $\beta$ and Calreticulin in BAL or cell supernatants were measured by ELISA assay according to industrial instructions (R\&D systems, Minneapolis, MN).

\section{Flow Cytometry analysis}

$1 \times 10^{6}$ cell suspension from lung digests and BAL were incubated with antibody cocktail containing APCanti-CD206 (BioLegend. San Diego, CA), PerC-Cy5-anti-F4/80, PE-Cy7-anti-Ly6G, PE-Cy3-anti-p-STAT6, APC-Cy7-anti-CD11b, BV421-anti-Siglec F (BD Biosciences, Franklin Lakes, NJ and eBioscience, San Diego, CA). The cells stained with fluorescence-minus-one (FMO) antibody cocktail were unstained controls. The cells were incubated with antibod cocktail in PBS supplied with $3 \%$ FBS for 40 min and washed with PBS for 2 times at room temperature. Analysis was performed on FACScan cytometer and all data were analyzed using FlowJo software, version 8.8.4 (Becton, Dickinson and Company, Franklin Lakes, $\mathrm{NJ}$ ).

\section{Statistical analysis}


Results are presented as the mean \pm standard error of each group. All data were analyzed by Student's $t$ test for comparison between two groups and one-way analysis of variance (ANOVA) followed by Tukey's multiple comparisons test for over two groups. A value of $p<0.05$ was considered as statistically significantly different.

\section{Results}

\section{Lack of STAT6 expressioin in STAT6-/- mice induced more severe ALI}

STAT6 is involved in the regulation of macrophage biological function [9]. To investigate the role of STAT6 in the development of ALI, we established ALI mouse model by i.t. treatment of LPS in WT and STAT6-/- mice. 2 days after the treatment, we observed the moderately increased total STAT6 and pSTAT6 in the inflamed lung tissues, as measured by immunostaining analysis (Fig. 1A). Consistently, LPS treatment also increased p-STAT6 expression in the lung digests analyzed by flow cytometry analysis. However, p-STAT6 expression was largely reduced in PBS or LPS-treated STAT6-/- mice, indicating the STAT6 deficiency in STAT6-/- mice (Fig. 1B).

To further amalysis showed that lack of STAT6 induced more severe ALI in STAT6-/- mice than WT mice. The pathological score (Fig. 2A-B), total cell counts (Fig. 2C) and protein content (Fig. 2D) in BAL were significantly higher in the STAT6-/- mice than those in WT mice. In addition, we observed more expression of pro-inflammatory cytokines, including TNF-a, IL-6 and IL-1 $\beta$ in STAT6-/- mice compared to the WT mice $(p<0.05, n=7)($ Fig. 2E-G).

\section{Polarization of Siglec F + CD206 + M2 subtype macrophages was reduced in STAT6-/- mice}

As previously reported, macrophages were deivided into Siglec F-CD206- M1 and Siglec F + CD206 + M2 subtype macrophages [10]. To investigate whether lack of STAT6 expressioin in STAT6-/- mice affected polarization of the macrophage subtypes, we analayzed the expression of Siglec F and CD206 in F4/80(high)Ly6G(low) macrophages (MPs) in the lung tissues and BAL by flow cytometry analysis. The results revealed that the population of $\mathrm{F} 4 / 80$ (low) Ly6G(high) neutrophils (NPs) were significantly increased in the lung tissues and BAL of mice with LPS-induced ALI. However, lack of STAT6 expressioin in STAT6-/- mice induced 2-fold more NPs in BAL and lung tissues than WT controls (Fig. 3A-B). Due to massive influx of NPs into the lung tissues of mice with ALI, the percentage of MPs in lung tissues and BAL were relatively lower in STAT6-/- mice than those in WT mice. Further analysis of MP subtypes showed the increased Siglec F-CD206- M1 and but decreased Siglec F + CD206 + M2 subtype macrophages in WT mice with ALI. Lack STAT6 in STAT6-/- mice induced more M1, but lower M2 cells than thoses in WT mice (Fig. 3A, lower panel and C). The results indicated that lack of STAT6 promoted ALI, in association with the increased Siglec F-CD206- M1 and reduced Siglec F + CD206 + M2 subtype macrophages.

\section{Lack of STAT6 in STAT6-/- mice induced were more macrophage pyroptosis}

NLRP3 is a key protein component in pyroptotic cells. After cell activation, NLRP3 is up-regulated and coupled with adapter protein ASC and Caspase- 1 to form active NLRP3 inflammasome and secreting 
active inflammatory cytokines IL-1 $\beta$ and IL-18 $[13,14,17]$. However, it is unknown whether the increased ALI severity in STAT6-/- mice was associated with the enhanced macrophage pyroptosis. To address this issue, we analyzed the expression of NLRP3 and ASC in the inflamed lung tissues. The results of immunostaining and Western blot analysis revealed that lack of STAT6 expression significantly increased the expression of NLRP3 and ASC (Fig. 4A-C). In consistent with the results of NLRP3 and ASC expression in vivo, we observed more expression p-p38 MAPK at resideue Thr180 and Tyr182 in the lung tissues of STAT6-/- mice than those in WT mice (Fig. 4B-C). LC3A/B and p62 are important molecules in cell autophagy $[18,19]$. Our additional study by Western blot analysis also revealed the significantly increased expression of LC3A/B, but a trend of reduced p62 expression in the STAT6-/- mice, compared to those in WT mice (Fig. 4D-E). The results indicated that the increased ALI severity in STAT6-/- mice was associated with the enhanced macrophage pyroptosis.

\section{NLRP3 expression was increased in STAT6-/-bone marrow-derived macrophages (BMDMs)}

The pyroptotic macrophages exclusively expressed the high levels of NLRP3 and IL-1 $\beta$. To further define whether macrophage pyroptosis was involved in STAT6 deficiency-induced exacerbation of murine ALI in vitro, we treated BMDMs from WT and STAT6-/- mice with LPS for $24 \mathrm{hrs}$. Consistent with the results in vivo (Fig. 1A-B), both immunostaining and Western blot analysis showed the increased expression of $p$ STAT6 (Fig. 5A) and total STAT6 (Fig. 5B) in LPS-treated WT BMDMs. Additional Western blot analysis also showed further increases in the expression of both NLRP3 and p38 MAPK in STAT6-/- BMDMs, compared to those in LPS-treated WT BMDMs (Fig. 5B). Consistently, we observed the elevated expression of pro-inflammatory cytokines and mediators, including TNF-a, IL-1 $\beta$ and Calreticulin, but the reduced expression of anti-inflammatory IL-10 in the LPS-treated STAT6-/-BMDMs, compared to those in LPS-treated WT BMDMs (Fig. 5C). Therefore, lack of STAT6 expression enhanced LPS-treated macrophage pyroptosis, correlated to the more pro-inflammatory Siglec F-CD206- M1 subtype macrophages in STAT6-/- mice with ALI.

\section{Glibenclamide, PDTC and SB203580 reversed pyroptosis in STAT6-/- macrophages}

To further investigate whether lack of STAT6 drives macrophage pyroptosis through increasing NLRP3/p38 MAPK/NF-kappaB signaling pathway, we pre-treated WT or STAT6-/- BMDMs with NLRP3 inhibitor Glibenclamide (GLBM), NF-kappaB inhibitor PDTC and p38 MAPK inhibitor SB203580 (SB), respectively, one hour prior to LPS treatment. 24 hours after LPS treatment, we observed the moderately elevated CD80 expression in WT (data not shown) and STAT6-/- BMDMs. However, Glibenclamide, PDTC and SB203580 moderately reversed the LPS-induced up-regulation of CD80, with more suppressive effects of PDTC and SB203580 than Glibenclamide (Fig. 6A-B). Thereby, Glibenclamide, PDTC and SB203580 revsersed pyroptosis in STAT6-/- macrophages.

The results were further confirmed by measurement of IL-1 $\beta$ expression in supernatants of the treated cells, indicating the up-regulated expression of IL-1 $\beta$ in the LPS-treated STAT6-/- BMDMs and a trend of suppressed expression by pre-treatment with Glibenclamide, PDTC and SB203580 (Fig. 6C). The results were also consistent with the results by immunostaining analysis, in which LPS treatment increased the expression of NLRP3, Caspase-1 and ASC (red) in STAT6-/- BMDMs. However, the up-regulated NLRP3, 
Caspase- 1 and ASC were moderately down-regulated by Glibenclamide and PDTC pre-treatment in WT (data not shown) and STAT6-/-BMDMs (Fig. 6D). Thereby, NLRP3, NF-kappaB and p-p38 MAPK inhibitors effectively reversed macrophage pyroptosis in STAT6-/- macrophages. NLRP3, NF-kappaB and p-p38 MAPK were at downstream of STAT6 signaling. STAT6 may exert immune regulatory role by suppressing macrophage pyroptosis through suppressing downstream NLRP3/p38 MAPK/NF-kappaB signaling.

\section{Discussion}

Macropages pyroptosis is featured by activation of NLRP3 inflammasome and releasing a large amount of IL-18 and active IL-1 $\beta$. NLRP3 inflammasome is a protein complex, containing NLRP3, adaptor protein ASC and pro-IL-1 $\beta$ [20]. The pyroptotic cells have high expression of find-me signal, such as Calreticulin, that attracts live macrophages for clear-up [21]. It was previously reported that more expression of NLRP3, IL-18 and IL-1 $\beta$ was observed in ALI mouse model. If not effectively cleared, the increased pyroptotic macrophages and released cytokines may results in the uncontrolled lung inflammation among patients with ARDS [22]. Thus, suppression of pyroptotic macrophages through molecular intervention might be an effective therapeutic strategy in the treatment of the uncontrolled lung inflammation in ARDS patients [23-25].

It remains unclear how NLRP3 inflammasome is activated in macrophages. Pro-inflammatory microenvironment may contribute to the high expression of NLRP3, because previous report showed that NLRP3 expression can be up-regulated by TNF- $a$ and IL-6 [10]. Potassium ionophore nigericin and ATP are potent inducers of NLRP3 inflammasome [26]; whereas Glibenclamide, Rho-associated coiled-coil kinase inhibitor Y-27632, and Caspase-1 inhibitor can suppress the activation of NLRP3 inflammasome [16]. Therefore, NLRP3 inflammasome can be modulated by inflammatory stimuli and inhibitors. However, it is not well defined remains whether and how STAT6 signaling pathway is involved in the modulation of macrophage NLRP3 inflammasome and ALI in mice.

In a mouse model with ALI and LPS-treated macrophages, we observed lower expression and activation of STAT6, but high activation of NLRP3 inflammsome. The reduced JAK2/STAT6 pathway by LPS treatment was also previously reported in ovalbumin-induced asthma model [27]. To define whether macrophage pyroptosis contribute to the development of ALI through STAT6 signaling, we established ALI mouse model in WT and STAT6-/- mice. The results showed that lack of STAT6 in STAT6-/- mice induced more severe ALI, accompanied with more infiltration of neutrophils, indicating the immune regulatory role of STAT6 signaling in ALI mouse model. Further analysis of macrophage phenotypes also revealed more population of Siglec F-CD206- subtype M1 macrophages, but lower Siglec F + CD206 + subtype M2 macrophages in the inflamed lung tissues and BAL. The results revealed the important role of STAT6 signaling in promoting M2 subtype macrophage polarization in ALI mouse, consistent with the previous reports $[28,29]$. Thus, we conclude that lack of STAT6 in STAT6-/- mice increased M1-biased polarization of macrophages and severity of ALI. The high expression of pro-inflammatory cytokines, including TNF- $\alpha$ and IL-1 $\beta$ from M1 subtype macrophages may accounts for the debilitating effects [30]. 
In addition to the increasing M1 cell-biased polarization, we also observed more pyroptotic cells in STAT6-/- mice with ALI. There was more expression of NLRP3 and ASC in the lung tissues of STAT6-/mice than WT mice. The increased pyroptosis was associated with the elevated expression of IL-1 $\beta$ from macrophages. Thereby, lack of STAT6 increased ALI severity, possibly through increasing both M1-biased macrophage polarization and pyroptosis in the inflamed lung tissues. Because STAT6 signaling is also involved in phagocytosis activity [6], we speculate that the effective clear-up of pyroptotic macrophages, apoptotic neutrophils and other dead cells may be compromised in STAT6-/- mice and that subsequently results in more severe lung inflammation in ALI mice.

Taken together, we conclude that lack of STAT6 induced more severe ALI in mice, associated with the increased macrophage pyroptosis and autophagy. However, it remains uncelar what downstream signaling pathways were involved in the debilitating effects. Previous reports demonstrated that p38 MAPK and NF-KB signaling participated in LPS-induced activation of NLRP3 inflammasome [22, 31]. Consistent with the previous reports [10], we also observed the increased expression of p-p38 MAPK in the LPS-induced mouse model and macrophages, suggesting the critical role of STAT6 signaling in suppression of p38 MAPK activation. LPS may activate p38 MAPK though suppressing STAT6 signaling. However, the relationship between p38 MAPK and STAT6 signaling is controversial. It was reported that IL-13 suppressed p38 MAPK activation through activation of STAT6 signaling in the intestine epithelial cells, implying that STAT6 signaling suppressed p38 MAPK activation [31]. However, it was also reported that IL-13 can not activate p38 MAPK in STAT6-/- mouse tracheal epithelial cells, implying that IL-13 may activate p38 MAPK through STAT6 signaling [32]. The discrepancy maybe caused by different cell types and animal models. Because NLRP3/p38 MAPK/NF-kappaB signaling is involved in macrophage pyroptosis, our additional studies in vitro showed that NLRP3, NF-kappaB and p38 MAPK inhibitors can effectively suppress LPS-induced activation of STAT6-/- macrophage pyroptosis. Thus, NLRP3/p38 MAPK/NF-kappaB signaling is at downstream of STAT6 signaling.

In summary, lack of STAT6 enhanced the developemt of mice with ALI, associated with more polarization of Siglec F-CD206- subtype macrophages, increased macrophage pyroptosis. STAT6 signaling plays the immune regulatory role by suppressing downstream NLRP3/p38 MAPK/NF-kappaB signaling, that accounts for the suppressed polarization of Siglec F-CD206- subtype macrophages. Molecular intervention of STAT6 signaling would be a potential approach in the treatment of ALI/ARDS.

\section{Abbreviations}

ALI, murine acute lung injury; ARDS, acute respiratory distress syndrome; ASC, apoptosis-associated speck-like protein containing a CARD; BAL, bronchoalveolar lavage; BMDMs, bone marrow-derived macrophages; GAPDH, glyceraldehyde-3-phosphate dehydrogenase; KO, knock-out; LPS, lipopolysaccharides; M2 cells, alternatively activated macrophages; MPs, macrophages; NLRP3, Nod-like receptor containing a pyrin domain 3; NPs, neutrophils; WT, wild-type.

\section{Declarations}


Acknowledgments: This study was supported by research grant from the Natural Science Foundation of Shanghai (19ZR1409000) and Fudan University Zhongshan Hospital Foundation in China (A654116001) to ZJ.

Author's contributions: HU L, Shao CZ and Pan LY participated in Western blot analysis. Jiang Z participated in the generation of hypothesis, animal and cell experiments, data analysis and assembly, manuscript writing, revision, and is responsible for all direction of the work. All authors read and approved the final manuscript.

Consent to participate: All authors consent to participate in this study

Consent for publication: All authors consent to publish the content of this study

Availability of data and materials: Data and materials are available upon request.

\section{Compliance with Ethical Standards}

Declarations: The authors declare no conflict of interest.

Ethics approval: The protocol was reviewed and approved by the Institutional Animal Care and Use Committee of the Fudan University, Zhongshan Hospital in China.

\section{References}

1. Chen, G., et al. 2018. Inhibition of CRTH2-mediated Th2 activation attenuates pulmonary hypertension in mice. The Journal of experimental medicine 215, 2175-2195.

2. Symowski, C. \& Voehringer, D. 2019. Th2 cell-derived IL-4/IL-13 promote ILC2 accumulation in the lung by ILC2-intrinsic STAT6 signaling in mice. European journal of immunology 49, 1421-1432.

3. Srinivasa, B.T., et al. 2017. STAT6 inhibitory peptide given during RSV infection of neonatal mice reduces exacerbated airway responses upon adult reinfection. Journal of leukocyte biology 101, 519529.

4. Hall, S.L., et al. 2017. IL-17A enhances IL-13 activity by enhancing IL-13-induced signal transducer and activator of transcription 6 activation. The Journal of allergy and clinical immunology 139, 462471 e414.

5. Stokes, K., et al. 2015. Cutting edge: STAT6 signaling in eosinophils is necessary for development of allergic airway inflammation. Journal of immunology 194, 2477-2481.

6. Kim, M.J., et al. 2018. A STAT6 Inhibitor AS1517499 Reduces Preventive Effects of Apoptotic Cell Instillation on Bleomycin-Induced Lung Fibrosis by Suppressing PPARgamma. Cellular physiology and biochemistry : international journal of experimental cellular physiology, biochemistry, and pharmacology 45, 1863-1877. 
7. Long, M.E., et al. 2017. MEK1/2 Inhibition Promotes Macrophage Reparative Properties. Journal of immunology 198, 862-872.

8. Nepal, S., et al. 2019. STAT6 induces expression of Gas6 in macrophages to clear apoptotic neutrophils and resolve inflammation. Proceedings of the National Academy of Sciences of the United States of America 116, 16513-16518.

9. D'Alessio, F.R., et al. 2016. Enhanced resolution of experimental ARDS through IL-4-mediated lung macrophage reprogramming. American journal of physiology. Lung cellular and molecular physiology 310, L733-746.

10. Jiang, Z., Chen, Z., Hu, L., Qiu, L. \& Zhu, L. 2020. Calreticulin Blockade Attenuates Murine Acute Lung Injury by Inducing Polarization of M2 Subtype Macrophages. Frontiers in immunology 11, 11.

11. Simovic, M.O., Ballard, B.R., Gray, K.D. \& Stain, S.C. 2007. The STAT4 and STAT6 pathways in pancreatitis-associated lung injury. The Journal of surgical research 137, 10-15.

12. Wang, Q., et al. 2020. MCTR1 enhances the resolution of lipopolysaccharide-induced lung injury through STAT6-mediated resident M2 alveolar macrophage polarization in mice. Journal of cellular and molecular medicine.

13. de Almeida, L., et al. 2015. The PYRIN Domain-only Protein POP1 Inhibits Inflammasome Assembly and Ameliorates Inflammatory Disease. Immunity 43, 264-276.

14. Moretti, J. \& Blander, J.M. 2020. Increasing complexity of NLRP3 inflammasome regulation. Journal of leukocyte biology.

15. Hwang, l., et al. 2015. Non-transcriptional regulation of NLRP3 inflammasome signaling by IL-4. Immunology and cell biology 93, 591-599.

16. Tamura, K., et al. 2017. Glibenclamide inhibits NLRP3 inflammasome-mediated IL-1 beta secretion in human trophoblasts. Journal of pharmacological sciences 135, 89-95.

17. Gaidt, M.M., et al. 2016. Human Monocytes Engage an Alternative Inflammasome Pathway. Immunity 44, 833-846.

18. Li, L., et al. 2017. Klotho Regulates Cigarette Smoke-Induced Autophagy: Implication in Pathogenesis of COPD. Lung 195, 295-301.

19. Zhou, S., et al. 2018. Spermine Alleviates Acute Liver Injury by Inhibiting Liver-Resident Macrophage Pro-Inflammatory Response Through ATG5-Dependent Autophagy. Frontiers in immunology 9, 948.

20. Khare, S., et al. 2012. An NLRP7-containing inflammasome mediates recognition of microbial lipopeptides in human macrophages. Immunity 36, 464-476.

21. Wang, Q., et al. 2013. Pyroptotic cells externalize eat-me and release find-me signals and are efficiently engulfed by macrophages. International immunology 25, 363-372.

22. Li, D., Ren, W., Jiang, Z. \& Zhu, L. 2018. Regulation of the NLRP3 inflammasome and macrophage pyroptosis by the p38 MAPK signaling pathway in a mouse model of acute lung injury. Molecular medicine reports $18,4399-4409$. 
23. Shao, X.F., et al. 2020. Ghrelin alleviates traumatic brain injury-induced acute lung injury through pyroptosis/NF-kappaB pathway. International immunopharmacology 79, 106175.

24. Wang, Y.C., et al. 2019. Dihydromyricetin Alleviates Sepsis-Induced Acute Lung Injury through Inhibiting NLRP3 Inflammasome-Dependent Pyroptosis in Mice Model. Inflammation 42, 1301-1310.

25. Xu, W.J., et al. 2019. Inhibition of GGPPS1 attenuated LPS-induced acute lung injury and was associated with NLRP3 inflammasome suppression. American journal of physiology. Lung cellular and molecular physiology 316, L567-L577.

26. Accarias, S., et al. 2015. Pyroptosis of resident macrophages differentially orchestrates inflammatory responses to Staphylococcus aureus in resistant and susceptible mice. European journal of immunology 45, 794-806.

27. Ding, F., et al. 2018. LPS Exposure in Early Life Protects Against Mucus Hypersecretion in OvalbuminInduced Asthma by Down-Regulation of the IL-13 and JAK-STAT6 Pathways. Cellular physiology and biochemistry : international journal of experimental cellular physiology, biochemistry, and pharmacology 46, 1263-1274.

28. Goenka, S. \& Kaplan, M.H. 2011. Transcriptional regulation by STAT6. Immunol Res 50, 87-96.

29. Gordon, S. \& Martinez, F.O. 2010. Alternative activation of macrophages: mechanism and functions. Immunity 32, 593-604.

30. Jiang, Z. \& Zhu, L. 2016. Update on the role of alternatively activated macrophages in asthma. J Asthma Allergy 9, 101-107.

31. Dames, P., et al. 2015. Interleukin-13 affects the epithelial sodium channel in the intestine by coordinated modulation of STAT6 and p38 MAPK activity. The Journal of physiology 593, 52695282.

32. Fujisawa, T., et al. 2008. Involvement of the p38 MAPK pathway in IL-13-induced mucous cell metaplasia in mouse tracheal epithelial cells. Respirology 13, 191-202.

\section{Figures}


A. Lung

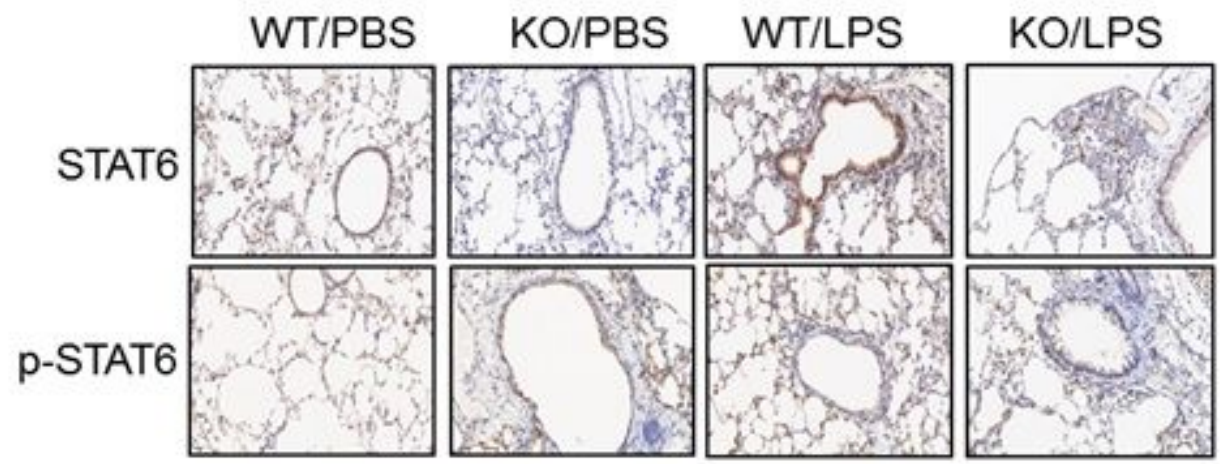

B

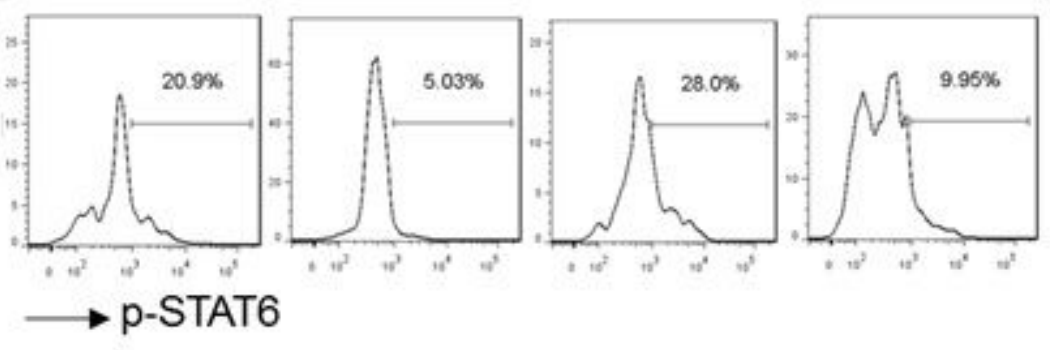

Figure 1

\section{Figure 1}

LPS increased STAT6 expression in the lung tissues of mice with ALI. Wild-type (WT) and STAT6-/- adult mice with ALI were established by intratracheal (i.t.) administration of $5 \mathrm{mg} / \mathrm{kg}$ LPS for 2 days. PBS was administered as controls. (A) Immunostaining for total STAT6 and p-STAT6 expression in the lung sections of mice. The positively stained cells were developed by 3,3'-Diaminobenzidine (DAB, Brown). (B) Flow cytometry analysis of p-STAT6 expression in the lung digests of mice. Representative photograph and histogram of each group (A-B). 
A
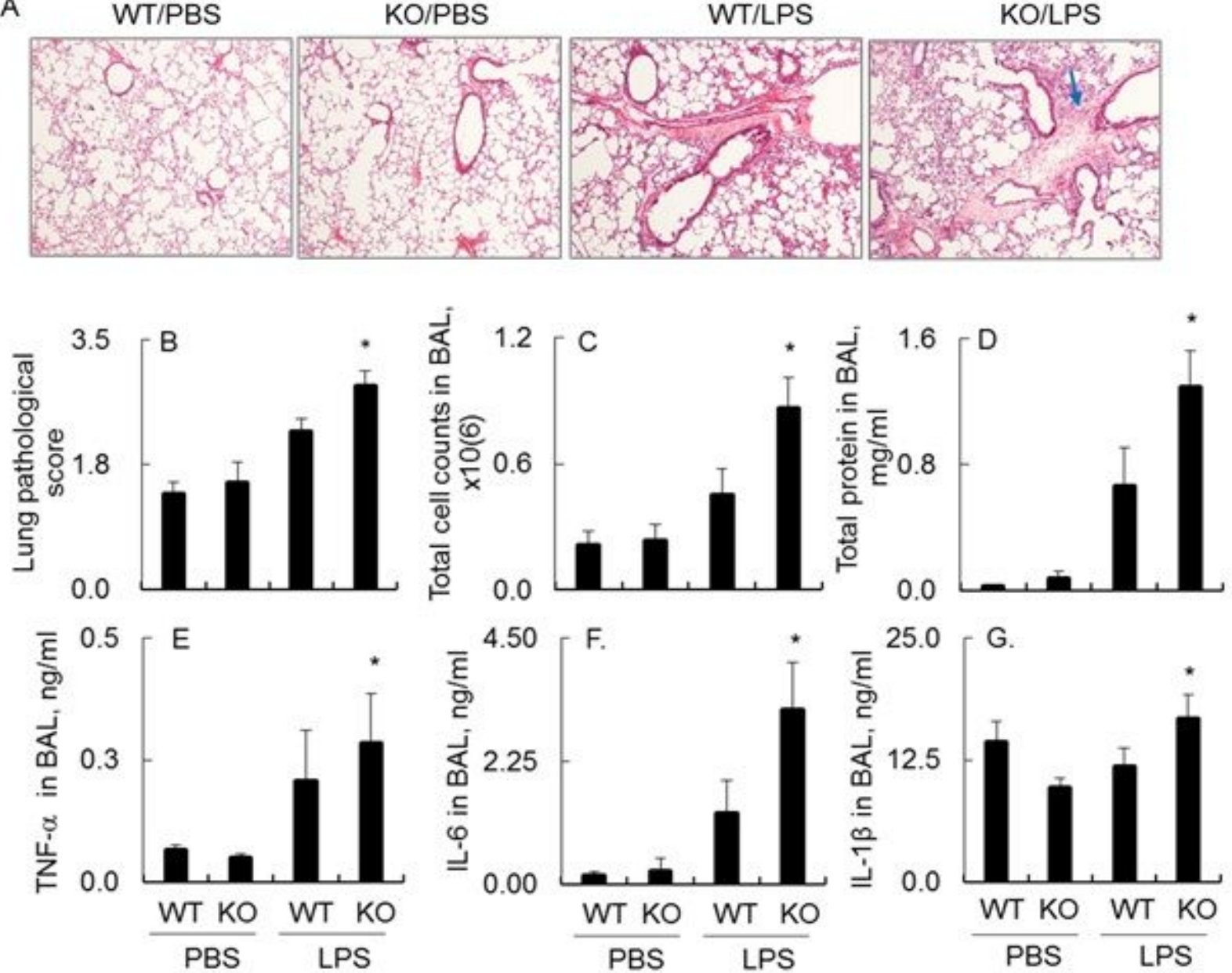

Figure 2

\section{Figure 2}

Lack of STAT6 expression in STAT6-/- mice induced more severe ALI. (A) Lung histology by H\&E staining. Representative photograph of each group. Blue arrow indicates the inflammatory infiltrates. (B) Quantitative analysis of lung inflammation in each group. The injury severity was evaluated by scale from 0 to 4 in terms of infiltrating inflammatory cells and alveoli destruction. (C) Total cell counts in BAL. (D) Total protein content of each group by BCA assay. (E-G) ELISA analysis for TNF-叉, IL-6 and IL-1 $\beta$ in BAL. All quantitative data was presented as mean \pm standard error, * $p<0.05$ v.s. WT group, $n=7$. 
A. BAL WT/PBS
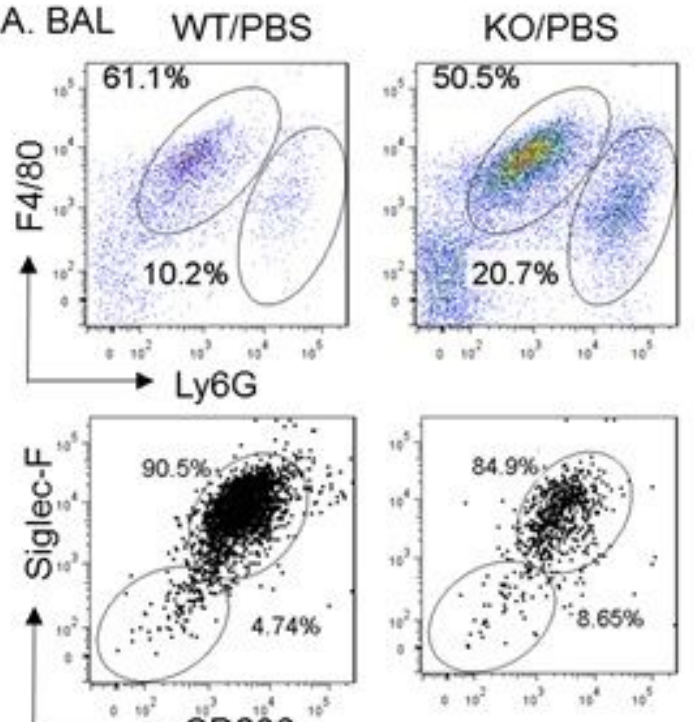

$\mathrm{CD} 206$
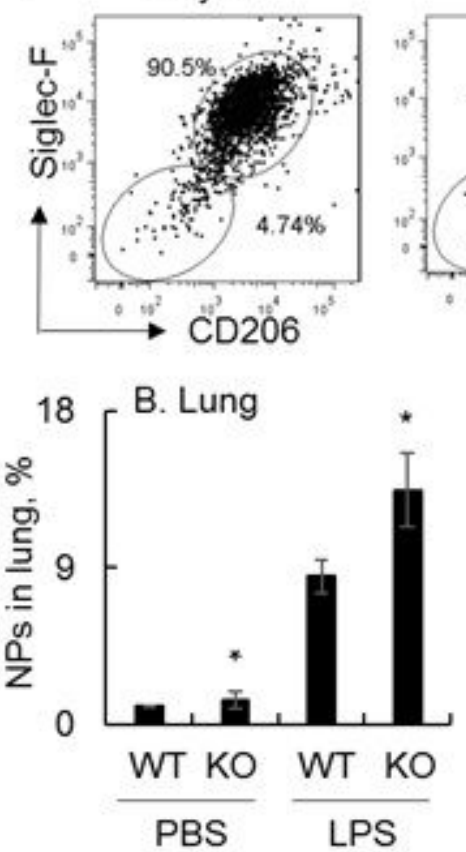

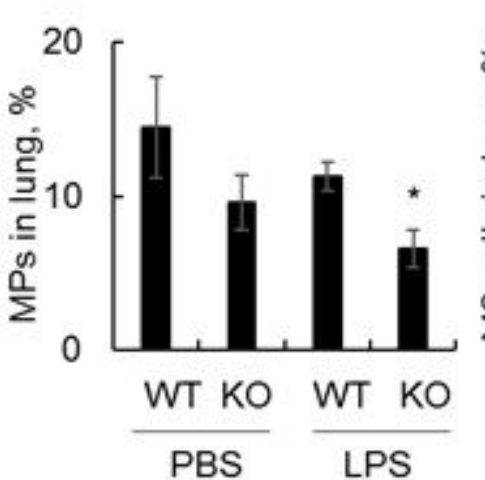

Figure 3
WT/LPS
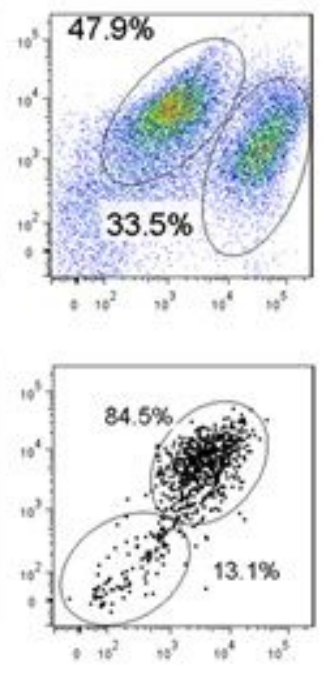

KO/LPS
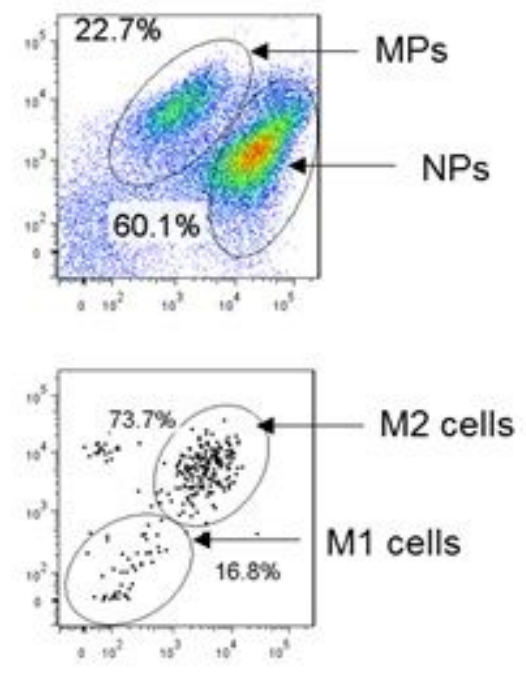

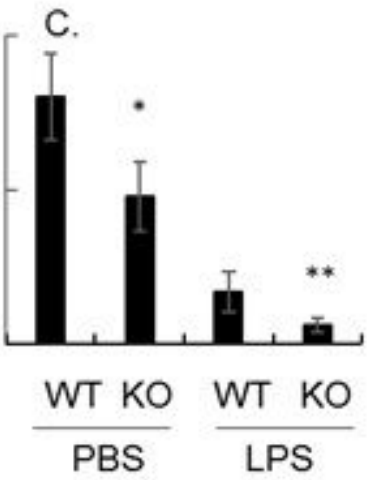

\section{Figure 3}

Lack of STAT6 expression in STAT6-/- mice attenuated polarization of Siglec F+CD206+ M2 subtype macrophages. (A) Flow cytometry analysis for F4/80(low)Ly6G(high) neutrophils (NPs) and F4/80(high)Ly6G(low) macrophages (MPs) in BAL of mice treated with LPS or PBS controls (upper panel). Siglec F-CD206- M1 and Siglec F+CD206+ M2 subtype macrophages were gated on MPs (lower panel). Representative dot plot was shown in each group. (B) Quantitative analysis of NPs and MPs in the lung tissues of mice in each group. (C) Quantitative analysis of M2 subtype macrophages in the lung tissues. All quantitative data was presented as mean \pm standard error, ${ }^{\star} p<0.05,{ }^{\star \star} p<0.01$ v.s. WT group, $\mathrm{n}=7$. 

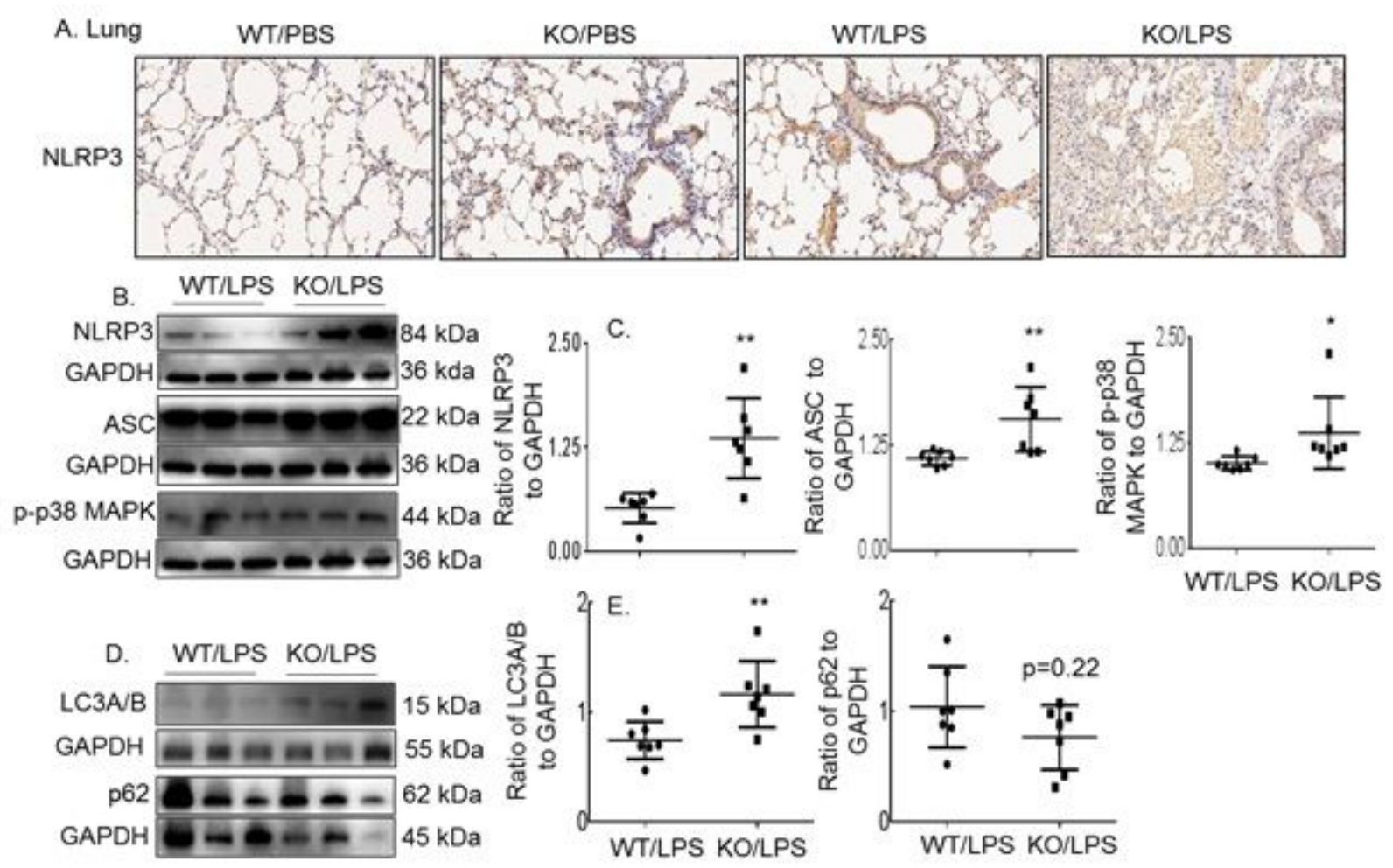

Figure 4

\section{Figure 4}

Lack of STAT6 expression in STAT6-/- mice induced more cell pyroptosis. (A) Immunostaining for NLRP3 expression in the lung tissues of mice with ALI. Representative photograph was shown. Yellow indicates positively stained cells. (B) Western blot analysis for protein expression of NLRP3, ASC and p-p38 MAPK in the lung tissues of mice with ALI. Each lane indicates individual mouse sample. GAPDH is internal controls. (C) Quantitative analysis of NLRP3, ASC and p-p38 MAPK expression by Western blot. (D) Western blot analysis for protein expression of LC3A/B and p62 in the lung tissues of mice with ALI. Each lane indicates individual mouse sample. (E) Quantitative analysis of LC3A/B and p62 expression by Western blot. Data was presented as dot plot. ${ }^{*} p<0.05,{ }^{*} p<0.01$ v.s. WT/LPS group. 

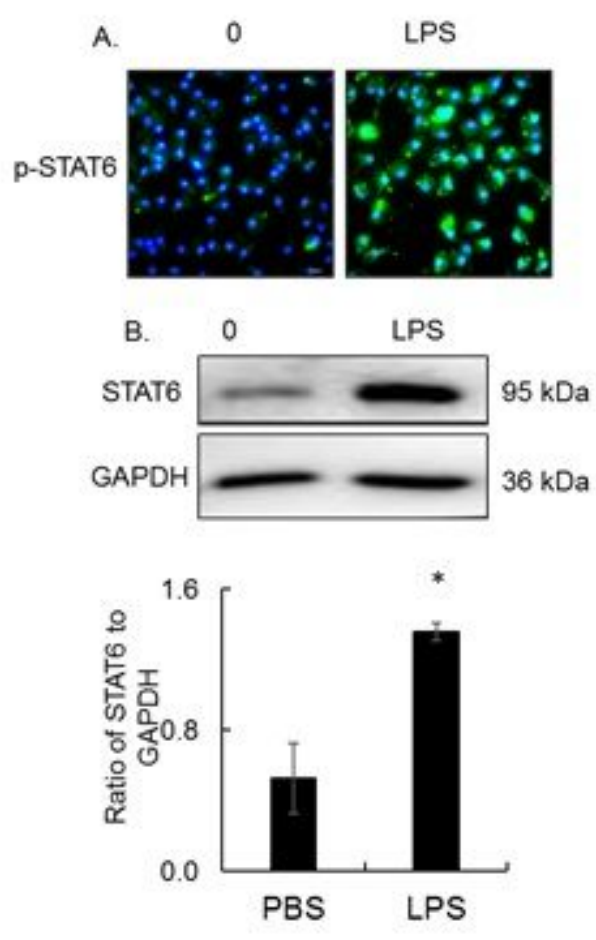
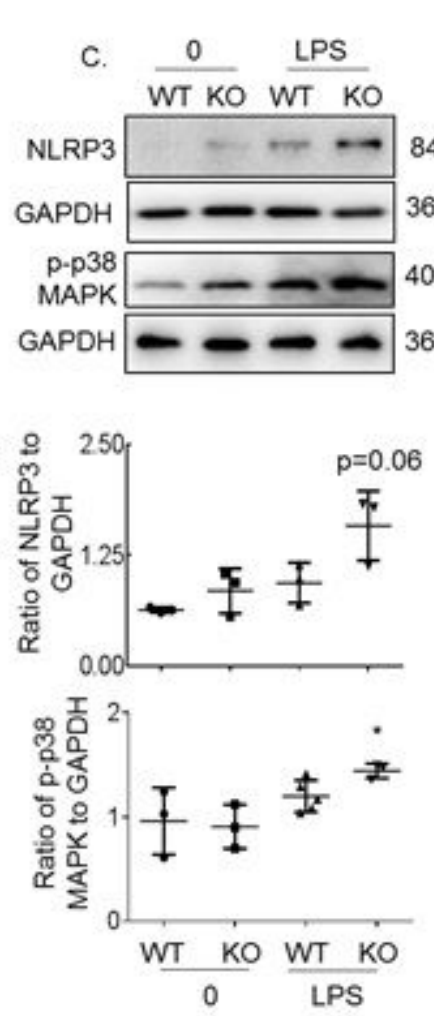

Figure 5
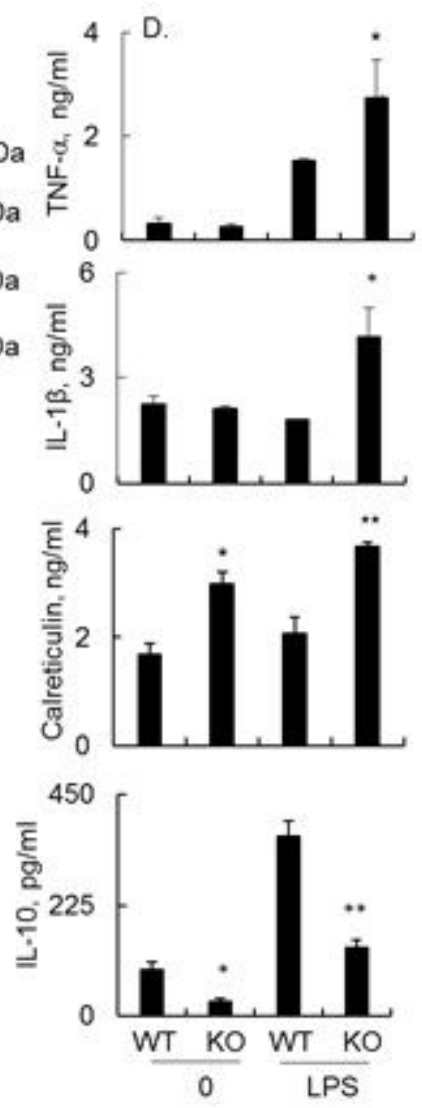

\section{Figure 5}

Lack of STAT6 increased the expression of NLRP3 and IL-1 $\beta$ in bone marrow-derived macrophages (BMDMs). (A) Immunostaining analysis for the expression of p-STAT6 in WT BMDMs. BMDMs were untreated or treated with $500 \mathrm{ng} / \mathrm{ml}$ LPS for 24 hours. Representive photograph was shown. (B) Western blot analysis for the expression of total STAT6 in the LPS-treated BMDMs. Representive photograph was shown (upper panel). Quantitative analysis of total STAT6 (lower panel). $n=3,{ }^{*} p<0.05$. (C) Western blot analysis for the expression of NLRP3, p-p38 MAPK in the LPS-treated WT and STAT6-/- BMDMs (upper panel). Quantitative analysis of NLRP3 and p-p38 MAPK (lower panel). $n=3,{ }^{*} p<0.05$ v.s. WT cells. (D) ELISA analysis for the expression of TNF- $\mathrm{Z}, \mathrm{IL}-1 \beta$, Calreticulin and IL-10 in the supernatants of treated cells. Data was presented as mean \pm standard error, $n=3,{ }^{*} p<0.05$, ${ }^{*} p<0.01$ v.s. WT cells. 